\title{
Surface imaging of HD 199178 (V1794 Cygni)^
}

\author{
T. Hackman ${ }^{1}$, L. Jetsu ${ }^{1}$, and I. Tuominen ${ }^{2}$ \\ 1 Observatory, PO Box 14, 00014 University of Helsinki, Finland \\ 2 Astronomy Division, PO Box 3000, 90014 University of Oulu, Finland
}

Received 13 September 1999 / Accepted 10 May 2001

\begin{abstract}
We present surface temperature maps for the FK Comae-type star HD 199178 (V1794 Cygni) calculated from high resolution spectra obtained in 1994 and 1995. The spot pattern evolves, but all maps reveal a large cool spot remaining nearly at the same high latitude. The main spot is $1200-1600 \mathrm{~K}$ cooler than the mean surface temperature. The observed slightly flat bottomed absorption lines would usually be interpreted as evidence for a large cool polar spot. We argue that antisolar surface differential rotation offers a better explanation for the box like shape of the line profiles. However, we do not find conclusive evidence for antisolar differential rotation and note that there are still other possible explanations for the slightly flat bottomed line profiles.
\end{abstract}

Key words. stars: activity, imaging, late-type, starspots, individual: HD 199178

\section{Introduction}

Doppler or surface imaging is, at present, the most powerful tool to map spots on rapidly rotating late-type stars. Even so, the Doppler imaging maps should always be studied critically. The method suffers from difficulties in modeling spectral lines in late-type stars, as well as from uncertainties in stellar and spectral parameters. These problems have been amply illustrated by conflicting results. For instance, the temperature maps obtained with some Doppler imaging methods most often show very large cool spots covering the poles (cf. Vogt 1988; Strassmeier 1994; Hatzes \& Vogt 1992), while the results with the methods developed by Piskunov (1991) and Berdyugina (1998) rarely show polar spots (cf. Piskunov et al. 1990; Strassmeier et al. 1991; Korhonen et al. 2000). Since partly the same stars, and once even the same observations (Strassmeier et al. 1991), have been modeled, it is evident that the results depend either on the applied methods or the selected stellar and spectral parameters.

There are several studies on how line and stellar parameters influence Doppler imaging (cf. Unruh \& Collier Cameron 1995). But the influence of one important

Send offprint requests to: T. Hackman, e-mail: Thomas.Hackman@Helsinki.Fi

* Based on observations made with the $2 \mathrm{~m}$ telescope of the National Astronomical Observatory, Rozhen, Bulgaria, and the Nordic Optical Telescope, operated on the island of La Palma jointly by Denmark, Finland, Iceland, Norway, and Sweden, in the Spanish Observatorio del Roque de los Muchachos of the Instituto de Astrofisica de Canarias. phenomenon, namely surface differential rotation, has usually been neglected. Many active late-type stars show clear variations in their photometric rotation periods (Strassmeier et al. 1989; Cutispoto 1998). Variable periods have also been discovered in the Ca II H\&K emission (Donahue et al. 1996). These periodicity changes are usually explained as a signature of surface differential rotation combined with latitudinal activity migration. Apart from distorting the spot pattern, differential rotation would alter the shape of the line profile in the same way as a large cool or hot polar spot. This emphasizes the necessity of implementing surface differential rotation into the Doppler imaging techniques.

The generally accepted empirical result is that differential rotation is weaker in more rapidly rotating stars (Hall 1991). Hence the effects of differential rotation have usually been bypassed in Doppler imaging. Although antisolar differential rotation has previously been noted to alter the line profiles in a similar way as a large cool polar spot, Hatzes et al. (1996) concluded that this would require an unreasonably strong differential rotation.

HD 199178 (V1794 Cygni) is a rapidly rotating single G5 subgiant (Herbig 1958), which was classified as a FK Comae-type star by Bopp \& Rucinski (1981). Jetsu et al. (1990) derived a photometric rotation period of $P_{\text {phot }}=3$. $337484 \pm 0.000043$. Furthermore, they reported a 9.1 year period in the mean brightness level and indications of a shorter cycle in the light curve amplitude. In a recent analysis of photometry between 1975 and 1995 , Jetsu et al. (1999b) showed that the photometric period 
varied and interpreted this as a signature of differential rotation.

We present new temperature maps of HD 199178 for the years 1994 and 1995. The maps were calculated with the surface imaging technique developed by Piskunov (1991). Previous Doppler images of this star display a large cool polar spot and some smaller high and low latitude features (Vogt 1988; Strassmeier et al. 1999). Unlike these earlier studies of HD 199178, we take into account the possible effect of differential rotation on the rotational broadening of photospheric absorption lines. We also demonstrate how different values for the differential rotation coefficient alter the retrieved surface imaging map.

\section{Stellar parameters of HD 199178}

HD 199178 has an apparent visual magnitude of $V \approx 7{ }^{\mathrm{m}} 2$. The Hipparcos-satellite parallax measurement of $\pi \approx$ $10.68 \pm 0.73$ mas gives a distance of 94 pc (Perryman 1997). The long-term mean colour indices $B-V \approx 0.79$ and $V-R \approx 0.68$ determined by Jetsu et al. (1990) suggested an average "background" stellar surface temperature of $T_{\text {eff }} \approx 5375 \mathrm{~K}$. The above results agree with the G5III-IV spectral classification by Herbig (1958).

The new Hipparcos distance measurement allows us to estimate the absolute magnitude and radius of the star. We use 7.09 as the apparent visual magnitude of the unspotted star (Strassmeier et al. 1999). HD 199178 lies in the direction of the North America and Pelican Nebulae complex, but in the foreground. Assuming an interstellar absorption of 0.2 (Straižys et al. 1989: Fig. 2) yields an absolute visual magnitude of 2 m 02 . A bolometric correction of $B C=-0.184$ (Strassmeier et al. 1999) gives the bolometric magnitude $M_{\mathrm{bol}} \approx 1 \mathrm{~m} 84$. Finally, if the unspotted temperature of $T_{\text {eff }}=5400 \mathrm{~K}$ is combined to the solar $T_{\odot}=5800 \mathrm{~K}$ and $M_{\mathrm{bol}_{\odot}}=4 .{ }^{\mathrm{m}} 64$, the Stefan-Boltzmann relation gives $R \approx 4.2 R_{\odot}$.

The Barnes-Evans relation is based on the same principle as the above, except that the value of the bolometric correction is not required. We use the formulation by Lacy (1977):

$\log \left(R / R_{\odot}\right)=7.4724-0.2 V_{0}-2 F_{\mathrm{v}}+\log d$,

where $F_{\mathrm{v}}=3.977-0.429(V-R)_{0}$ and $[d]=$ pc. The combination $V_{0}=7{ }^{\mathrm{m}} 0, V-R=0.68$ and $E_{V-R}=0.05$ gives $F_{\mathrm{v}} \approx 3.707$ and $R \approx(4.3 \pm 0.7) R_{\odot}$. (Here $V_{0}$ is the mean apparent visual magnitude without interstellar absorption.)

The rotation period $P \approx 3^{\mathrm{d}} 3$ and projected rotational velocity $v \sin i \approx 70 \mathrm{~km} \mathrm{~s}^{-1}$ give a lower limit $R \sin i \approx$ 4.6 $R_{\odot}$ for the radius. There is thus a slight discrepancy between the different radius determinations. Anyway, the above estimates and the lower limit $R \sin i$ suggest that $4.6 R_{\odot} \lesssim R \lesssim 5.0 R_{\odot}$. This is equivalent to an inclination of $67^{\circ} \lesssim i \lesssim 90^{\circ}$. The exceptional nature of HD 199178 (rapid rotation, spots, possibly a coalesced binary) may mean that the equatorial radius will be underestimated.
In our calculations we will thus allow for an inclination $i \geq 50^{\circ}$.

A variable photometric rotation period was detected in the long-term photometry by Jetsu et al. (1999b). They analyzed 114 sets of photometric data obtained between 1975 and 1995. Each set contained at least 8 nights of observations and had a length of about 30 nights (Jetsu et al. 1999a). The regular yearly change rate of the photometric period was $3.3 \%$. In addition, irregular seasonal changes of $7.5 \%$ were found. The varying photometric period was explained by surface differential rotation quantified by $|\alpha| \gtrsim 0.075$, where

$\alpha=\left(\Omega_{\text {equator }}-\Omega_{\text {pole }}\right) / \Omega_{\text {equator }}$.

Even this lower limit for the differential rotation is considerably larger than that expected from the results by Hall (1991). If the latitudinal range of the spot activity does not extend all the way from the equator to the pole, then $|\alpha|$ could even reach the solar value of 0.2 . We thus assume that the realistic differential rotation coefficient values are within the limits $-0.20 \leq \alpha \leq 0.20$.

Differential rotation would alter the shape of the line profile. The case of $\alpha>0$ induces a "sharper" profile, while $\alpha<0$ causes a more "flat bottomed" profile (cf. Bruning 1981). Thus a negative $\alpha$ modifies the line profile much in the same way as a cool polar spot.

Since there is no unique photometric period, in principle different periods could be used for different seasons. But this would make it harder to compare the surface images. Therefore we chose to use the mean period $P=3$. 3250 calculated for the years 1994 an 1995. Our ephemeris for the rotational phases was

$\mathrm{HJD}_{\min }=(2449511.150 \pm 0.070)+(3.3250 \pm 0.0012) E,(3)$

where the $\mathrm{HJD}_{\text {min }}$ is the epoch of the light curve minimum (Jetsu et al. 1999b).

\section{The surface imaging technique}

Spots on the surface of a rapidly rotating star will cause distortions ("bumps") in the observed spectral line profiles. As the star rotates, these bumps will move across the absorption line profiles. The main idea of surface imaging is to trace these distortions and retrieve a surface map of the star. We use the surface imaging technique developed by Piskunov (Piskunov et al. 1990, Eqs. (3-6) and (8); Piskunov 1991, Eqs. (5-8)), but also include Johnson $V$ photometry in the inversion.

Local line profiles and $V$-band fluxes are calculated for a set of temperatures and limb angles using stellar model atmospheres. This table is then used for the disk integration of a given surface temperature distribution. The surface imaging problem is solved by searching for a surface temperature distribution that minimizes the discprepancy between the observations and the calculated line profiles and light curve. 
Since we always have a confined temperature range of model atmospheres, the solution should be limited to temperatures between the minimum and maximum of the models $\left(T_{\min }\right.$ and $\left.T_{\max }\right)$. We do this by adding a "penalty function" to the quantity to be minimized. We use an integral of a double-sided Heaviside step function, which is nearly constant between $T_{\min }$ and $T_{\max }$, but grows nearly linearly when the temperature is below $T_{\min }$ or exceeds $T_{\max }$. Thus the penalty function merely prevents extreme temperatures in the solution.

The crucial point of surface imaging is how the line profiles for a given temperature distribution are calculated. Errors in the calculated line profiles will introduce artifacts in the resulting map and prevent the solution from reaching the observational signal-to-noise level. Errors in the line profiles can arise from numerous problems, e.g. wrong line parameters, missing lines, non-LTE effects, wrong limb darkening or inadequacies of the model atmospheres. The fact that the lines used for Doppler imaging are strong absorption lines, whose cores are formed high in the photosphere, puts extra demands on the line modeling.

The errors in the line profiles are not the only source of systematic errors. For example, an erroneous $v \sin i$ will manifest itself as a cool or hot belt in the image (cf. Berdyugina 1998). Errors in the microturbulence velocity will mainly change the average temperature of the solution. Doppler images are not sensitive to errors in the macroturbulence velocity (cf. Unruh \& Collier Cameron 1995). A wrong inclination will cause a latitudinal displacement and a slight distortion of the spot. Combined with surface differential rotation, the inclination will also influence the line profile. Decreasing the inclination will increase the effect of differential rotation on the line profile (cf. Bruning 1981).

Differential rotation will, of course, also change the spot distribution, since different latitudes have different rotational periods. In this study we do not take this effect into account. Firstly, differential rotation becomes important in this sense only if the locations of spots extend over a large latitude range. Secondly, we assume that the magnetic field strength is sufficient to resist the shearing effect of differential rotation within the spot(s).

Since it is not (yet) possible to calculate perfect line profiles, one can always expect some artifacts in Doppler images. Fortunately, most of these artifacts can easily be identified, since certain shapes of artifacts are related to particular error sources. The same error in the overall shape of all calculated line profiles may manifest itself as a spot band at a certain latitude (or a polar spot). However, if the rotational phase coverage is inadequate, this artifact might not be axisymmetric. Minor errors in the calculations (e.g. missing lines in blends) or the data reduction (e.g. remnants of cosmic spikes or uncertainty in the continuum normalization) may cause thin high contrast features, like vertical stripes, arches or even ovals. For example, the remains of a cosmic spike in one spectrum may cause a thin high contrast artifact at a stellar longitude corresponding to the wavelength of the spike and rotation phase of the observation. This in turn might cause additional hot and cold features to cancel out the effect of the artifact in the remaining observed phases. A missing line, on the other hand, may lead to an artifact repeated at each observed phase. Noise in the spectra might be interpreted as a series of small "bumps". Because such bumps will appear at arbitrary wavelengths, the resulting artifacts cannot be latitudinally confined, and will therefore be seen as vertical stripes in the image.

It is clear, that the retrieved longitude for the spots will usually be more reliable than the latitude. In some cases the longitude of the spot can be estimated even with a single spectrum, while the determination of the latitude requires a good phase coverage. Moreover, the latitude is sensitive to errors in the inclination and the general shape of the rotationally broadened line profile. The influence of all error sources is greatly reduced by using many spectral lines and simultaneous photometry in the inversion.

\section{Observations}

High resolution spectra of HD 199178 were measured in July 1994, August 1994, November 1994 and July 1995. The July 1994 data set was obtained with the $2 \mathrm{~m}$ RitcheyChretién telescope coudé-spectrograph of the National Astronomical Observatory (Rozhen), Bulgaria. The rest of the spectroscopic observations were made using the SOFIN high resolution échelle spectrograph at the 2.5 m Nordic Optical Telescope (NOT), La Palma, Spain. The observed spectral regions were 6409-6414 $\AA$ (August 1994 and July 1995), 6427-6441 Å (all seasons) and 7509$7514 \AA$ (August 1994, November 1994 and July 1995). The typical signal-to-noise ratio was between 200 and 300 . The spectral observations are summarized in Table 1.

The spectral observations were reduced with the $3 \mathrm{~A}$ software system (Ilyin 2000). The reduction included bias, cosmic ray, flat field and scattered light correction, wavelength calibration and normalization. Special care was taken during the last two steps, where errors can cause artifacts in the surface images.

The calibration of the wavelength scale was obtained from Th-Ar comparison spectra. For the échelle spectra, we used all available spectral orders of the comparison frames to calculate a two-dimensional $(k \lambda)$ wavelength solution, taking into account distortions of the line positions (Ilyin 2000). Small shifts between the stellar and comparison spectra (e.g. due to flexure of the spectrograph) were corrected using atmospheric lines. The spectra were rebinned to a common wavelength grid.

The photospheric absorption lines of HD 199178 are broad and it is hard to determine the real continuum in the spectrum. Therefore the continuum normalization had to be done in two steps. A "quasi-continuum" consisting of points near to the real continuum level was used as a first approximation. The final normalization was done by utilizing a rotationally broadened synthetic spectrum. 
Table 1. The spectral observations of HD 199178: $t=$ HJD 2440000 for the middle of the exposures, rotational phases calculated from the ephemeris of Eq. (3), $S / N$ ratios and the resolution element $W_{\lambda}$.

\begin{tabular}{ccc|ccc}
\multicolumn{7}{l}{ July 1994, Rozhen, $W_{\lambda}=0.20 \AA$} \\
\hline$t$ & Phase & $S / N$ & $t$ & Phase & $S / N$ \\
\hline 9552.341 & 0.388 & 244 & 9559.479 & 0.535 & 212 \\
9552.457 & 0.423 & 268 & 9560.303 & 0.783 & 171 \\
9552.576 & 0.459 & 214 & 9560.474 & 0.834 & 320 \\
9553.309 & 0.679 & 219 & 9561.350 & 0.098 & 162 \\
9554.559 & 0.055 & 251 & 9562.353 & 0.399 & 286 \\
9555.421 & 0.315 & 251 & 9562.537 & 0.455 & 206 \\
9556.506 & 0.641 & 223 & 9563.352 & 0.700 & 236 \\
9557.537 & 0.951 & 248 & 9564.393 & 0.013 & 316 \\
9558.346 & 0.194 & 276 & 9565.381 & 0.310 & 263 \\
9558.561 & 0.259 & 178 & & & \\
\hline
\end{tabular}

August 1994, NOT-SOFIN/ 2nd camera, $W_{\lambda}=0.10 \AA$

\begin{tabular}{ccc|ccc}
\hline$t$ & Phase & $S / N$ & $t$ & Phase & $S / N$ \\
\hline 9578.544 & 0.269 & 209 & 9584.412 & 0.034 & 284 \\
9579.608 & 0.589 & 281 & 9585.409 & 0.334 & 238 \\
9580.521 & 0.863 & 277 & 9586.410 & 0.635 & 308 \\
9581.503 & 0.159 & 262 & 9587.441 & 0.945 & 263 \\
9582.418 & 0.434 & 246 & 9588.424 & 0.240 & 263 \\
9583.412 & 0.733 & 282 & & & \\
\hline
\end{tabular}

November 1994, NOT-SOFIN/ 1st camera, $W_{\lambda}=0.05 \AA$

\begin{tabular}{ccc|ccc}
\hline$t$ & Phase & $S / N$ & $t$ & Phase & $S / N$ \\
\hline 9669.355 & 0.580 & 150 & 9675.323 & 0.375 & 210 \\
9670.305 & 0.866 & 190 & 9676.322 & 0.676 & 210 \\
9671.412 & 0.199 & 180 & 9677.328 & 0.978 & 230 \\
9672.310 & 0.469 & 220 & 9678.325 & 0.278 & 250 \\
9673.309 & 0.770 & 210 & & & \\
\hline
\end{tabular}

July 1995, NOT-SOFIN/ 2nd camera, $W_{\lambda}=0.10 \AA$

\begin{tabular}{ccc|ccc}
\hline$t$ & Phase & $S / N$ & $t$ & Phase & $S / N$ \\
\hline 9910.599 & 0.135 & 295 & 9917.580 & 0.235 & 301 \\
9912.573 & 0.729 & 454 & 9918.588 & 0.538 & 321 \\
9913.598 & 0.037 & 332 & 9919.634 & 0.852 & 295 \\
9914.576 & 0.331 & 376 & 9920.623 & 0.150 & 319 \\
9915.616 & 0.644 & 325 & 9921.532 & 0.423 & 362 \\
9916.628 & 0.948 & 281 & & & \\
\hline
\end{tabular}

Simultaneous or nearly simultaneous Johnson $V$ photometry for July 1994, August 1994 and July 1995 was obtained with the $60 \mathrm{~cm}$ telescope at the Mount Maidanak Observatory and the APT Phoenix-10 telescope (subsets $\mathrm{SET}=108,109$ and 111, Jetsu et al. 1999a).

\section{Temperature maps}

The following three spectral regions were used for surface imaging: 6409.0-6413.5 $\AA$ (strongest line Fe I $\lambda 6411.649 \AA$ ), 6427.5-6441.0 ^ (Fe I $\lambda 6430.846 \AA$;
Fe II $\lambda 6432.680 \AA$ A; Ca I $\lambda 6439.075 \AA)$ and $7509.0-$ $7513.5 \AA$ (Fe I $\lambda 7511.020 \AA)$. The local line profiles were calculated for 20 limb angles and temperatures 3500$6500 \mathrm{~K}$ (with a step of $250 \mathrm{~K}$ ) using a code written by Berdyugina (1991) and atmospheric models by Kurucz (1993) with solar element abundances. Spectral line parameters were obtained from the Vienna Atomic Line Database (Kupka et al. 1999). All significant atomic lines and the most important molecular lines ( $\mathrm{CN}$ and $\mathrm{TiO}$ ) were included in the calculations. The final adjustment of the $g f$ values was done after analyzing the wavelength dependence of the deviation between a synthetic spectrum of HD 199178 and the mean of the observations. Of the 111 lines used in the calculations, the $g f$ value of 36 lines was changed. The changes in the strongest lines, Fe I $\lambda 6411.649 \AA$, Fe I $\lambda 6430.846 \AA$, Fe II $\lambda 6432.680 \AA$, CaI $\lambda 6439.075 \AA$ and Fe I $\lambda 7511.020 \AA$, were $-0.1<$ $\Delta \log (g f)<0.02$. It should be emphasized that the adopted $g f$ values may be biased by uncertainties in element abundances. A list of all atomic lines that we used, can be found in Table 2 .

The temperature maps were derived using the INVERS7-9PD code, originally written by N. E. Piskunov, with some changes made by T. Hackman. We first tested different values of rotational velocity (68 $\left.\mathrm{km} \mathrm{s}^{-1} \leq v \sin i \leq 74 \mathrm{~km} \mathrm{~s}^{-1}\right)$, microturbulence $\left(1.2 \mathrm{~km} \mathrm{~s}^{-1} \leq v_{\text {micro }} \leq 1.8 \mathrm{~km} \mathrm{~s}^{-1}\right)$, inclination $\left(50^{\circ} \leq\right.$ $\left.i \leq 85^{\circ}\right)$ and surface differential rotation $(-0.20 \leq \alpha \leq$ $0.20)$. Differential rotation was implemented by adjusting the angular rotational velocity at each latitude $b$ using the Maunder rotation formula

$\Omega(b)=\Omega_{\text {equator }}\left(1-\alpha \sin ^{2} b\right)$.

The best solution (the smallest deviation between the spectroscopic observations and the calculated line profiles) was obtained with the stellar parameters given in Table 3.

Before combining all available spectral regions, separate images were calculated for each line blend in order to check for inconsistencies. The individual maps for each spectral region gave the same main features, but contained more artifacts (small high contrast features, especially at low latitudes) than the maps obtained from the combination of all spectral regions.

All surface images show a large cool spot remaining at approximately the same latitude (Figs. 1-4 and Table 4). The mean temperature of the visible surface is $5300 \mathrm{~K}$. The polar region is about $4900 \mathrm{~K}$. The lower latitude features in the images are probably artifacts. In all maps there are symmetry effects typical for surface imaging of stars with relatively high inclination $\left(i \geq 60^{\circ}\right)$, i.e. "shadows" of the high latitude spot below the equator. The bright features, reaching a maximum of $T_{\text {eff }} \approx 5800 \mathrm{~K}$, are probably also artifacts.

The July 1994 image shows a large high latitude cool spot $(3700 \mathrm{~K})$ with a longitudinal appendage $(3900 \mathrm{~K})$. In the August 1994 image, the coolest part of the spot $(3700 \mathrm{~K})$ is more concentrated and the appendage (4200 K) is weaker. In November 1994, the spot appears 
Table 2. Atomic lines, lower excitation potential and adopted $g f$ values.

\begin{tabular}{|c|c|c|c|c|c|c|c|c|c|c|c|}
\hline Line & $\lambda(\AA)$ & $\chi_{\text {low }}(\mathrm{eV})$ & $\log (g f)$ & Line & $\lambda(\AA)$ & $\chi_{\text {low }}(\mathrm{eV})$ & $\log (g f)$ & Line & $\lambda(\AA)$ & $\chi_{\text {low }}(\mathrm{eV})$ & $\log (g f)$ \\
\hline Si I & 6407.070 & 5.871 & -2.200 & Sm I & 6425.905 & 0.282 & -1.815 & Ti I & 6435.315 & 2.250 & -2.875 \\
\hline Zr I & 6407.216 & 0.154 & -2.700 & Si I & 6426.291 & 5.954 & -2.470 & V I & 6435.490 & 2.684 & -1.582 \\
\hline Fe II & 6407.251 & 3.889 & -3.699 & Sm II & 6426.628 & 1.746 & -0.559 & Ce I & 6436.399 & 0.467 & -0.822 \\
\hline Si I & 6407.291 & 5.871 & -1.500 & Zr I & 6426.706 & 2.746 & -0.620 & $\mathrm{Fe} I$ & 6436.407 & 4.186 & -2.360 \\
\hline Co I & 6407.491 & 4.395 & -1.471 & Si I & 6426.911 & 5.954 & -2.900 & Y I & 6437.169 & 2.294 & -0.520 \\
\hline $\mathrm{Fe} I$ & 6407.643 & 4.076 & -3.620 & Sm II & 6428.355 & 1.370 & -1.150 & Ti I & 6437.610 & 3.407 & -1.889 \\
\hline $\mathrm{Fe} I$ & 6408.018 & 3.686 & -1.018 & $\mathrm{Nd}$ II & 6428.645 & 0.205 & -1.831 & Eu II & 6437.640 & 1.320 & 0.427 \\
\hline S I & 6408.157 & 7.868 & -1.510 & $\mathrm{Ca} \mathrm{I}$ & 6428.815 & 4.441 & -1.184 & Si I & 6437.703 & 5.863 & -2.210 \\
\hline $\mathrm{Fe} I$ & 6408.332 & 4.386 & -3.563 & Fe I & 6429.071 & 4.294 & -3.407 & Ni I & 6437.992 & 5.389 & -2.984 \\
\hline Sr I & 6408.459 & 2.271 & 0.500 & Pr II & 6429.629 & 1.615 & -0.386 & V I & 6438.088 & 2.684 & -2.070 \\
\hline Gd I & 6408.545 & 0.213 & -2.052 & $\mathrm{Nd} \mathrm{I}$ & 6429.840 & 1.337 & -0.530 & Cr I & 6438.586 & 3.890 & -2.571 \\
\hline Si I & 6408.671 & 5.984 & -3.090 & $\mathrm{Ni} \mathrm{I}$ & 6429.859 & 4.167 & -2.104 & Fe I & 6438.755 & 4.435 & -2.329 \\
\hline V I & 6408.814 & 2.616 & -1.992 & Co I & 6429.906 & 2.137 & -2.460 & $\mathrm{Ca} \mathrm{I}$ & 6439.075 & 2.526 & 0.450 \\
\hline Ti I & 6409.390 & 3.570 & -1.631 & Ca I & 6430.127 & 3.910 & -1.907 & Ti I & 6439.326 & 3.590 & -1.318 \\
\hline V I & 6410.416 & 2.581 & -1.889 & Co I & 6430.290 & 4.049 & -1.028 & Fe I & 6439.554 & 4.473 & -4.304 \\
\hline La I & 6410.975 & 0.373 & -0.950 & $\mathrm{Nb} \mathrm{I}$ & 6430.443 & 0.740 & -1.230 & Ti I & 6439.705 & 0.813 & -6.458 \\
\hline $\mathrm{ScI}$ & 6410.984 & 4.967 & -2.870 & V I & 6430.472 & 1.955 & -0.600 & Ce I & 6439.964 & 0.294 & -0.752 \\
\hline $\mathrm{Fe} I$ & 6411.107 & 4.733 & -1.905 & Ca I & 6430.793 & 3.910 & -2.129 & Si I & 6440.566 & 5.616 & -2.480 \\
\hline V I & 6411.276 & 1.950 & -2.059 & $\mathrm{Fe} \mathrm{I}$ & 6430.846 & 2.176 & -2.106 & $\mathrm{~N}_{\mathrm{I}}$ & 6440.938 & 11.764 & -1.140 \\
\hline Cr I & 6411.580 & 3.892 & -2.478 & Sm II & 6431.006 & 1.359 & -1.158 & Ti I & 6440.947 & 3.590 & -1.810 \\
\hline $\mathrm{Fe} I$ & 6411.649 & 3.654 & -0.645 & $\mathrm{Ca} \mathrm{I}$ & 6431.099 & 3.910 & -2.606 & Mn I & 6440.971 & 3.772 & -0.938 \\
\hline Co I & 6411.884 & 2.542 & -2.228 & V I & 6431.623 & 1.950 & -0.287 & $\mathrm{Fe} I$ & 6441.780 & 5.033 & -1.928 \\
\hline Ti I & 6412.192 & 3.581 & -1.480 & Pr II & 6431.805 & 1.416 & -0.339 & Si I & 6442.777 & 6.125 & -1.240 \\
\hline Fe I & 6412.200 & 2.453 & -4.441 & Sm II & 6431.978 & 1.413 & -1.460 & Fe II & 6442.955 & 5.549 & -2.885 \\
\hline Ti I & 6413.108 & 0.048 & -5.006 & Ni I & 6431.994 & 3.542 & -2.500 & $\mathrm{Fe} I$ & 7507.261 & 4.415 & -3.482 \\
\hline Sc I & 6413.324 & 0.021 & -2.677 & Er I & 6432.531 & 1.309 & -0.919 & $\mathrm{Fe} I$ & 7508.533 & 4.988 & -2.318 \\
\hline Ga I & 6413.468 & 3.073 & -0.300 & $\mathrm{Nd} \mathrm{I}$ & 6432.650 & 1.287 & -0.160 & $\mathrm{Fe} I$ & 7508.623 & 4.143 & -2.741 \\
\hline $\mathrm{C}_{\mathrm{I}}$ & 6413.550 & 8.771 & -1.380 & Fe II & 6432.680 & 2.891 & -3.540 & Ti I & 7509.728 & 1.749 & -3.030 \\
\hline Pr II & 6413.678 & 1.132 & -0.799 & $\mathrm{~V}_{\mathrm{I}}$ & 6433.162 & 1.945 & -1.308 & Si I & 7510.785 & 5.984 & -1.770 \\
\hline Mn I & 6413.945 & 3.763 & -1.910 & $\mathrm{Nb}$ I & 6433.204 & 0.657 & -1.480 & Fe I & 7511.020 & 4.178 & 0.059 \\
\hline Si I & 6414.188 & 6.083 & -2.450 & Si I & 6433.337 & 5.614 & -3.210 & $\mathrm{Nd}$ II & 7511.125 & 1.773 & -0.871 \\
\hline Ni I & 6414.581 & 4.154 & -1.220 & Si I & 6433.457 & 5.964 & -1.590 & Fe I & 7512.115 & 2.279 & -5.195 \\
\hline $\mathrm{Ni}$ I & 6414.796 & 4.165 & -2.463 & Fe II & 6433.814 & 6.219 & -2.371 & $\mathrm{Fe} I$ & 7512.139 & 4.143 & -2.947 \\
\hline Si I & 6414.980 & 5.871 & -1.100 & Zr I & 6434.388 & 2.798 & -0.090 & Cr II & 7512.745 & 4.756 & -2.630 \\
\hline $\mathrm{Fe} I$ & 6415.505 & 4.435 & -3.912 & Ce I & 6434.388 & 0.028 & -1.437 & Nd II & 7513.736 & 0.933 & -1.241 \\
\hline S I & 6415.522 & 7.870 & -1.360 & Y I & 6435.004 & 0.066 & -0.820 & Fe II & 7515.091 & 5.823 & -2.362 \\
\hline Nd II & 6425.779 & 1.649 & -0.935 & V I & 6435.158 & 1.942 & -1.458 & Fe II & 7515.831 & 3.903 & -3.432 \\
\hline
\end{tabular}

to be partly disintegrated. The coolest part of the spot (4100 K) seems to be at the position of the previous appendage. No photometric data was available for the November 1994 image, but there were clear changes in the light curves for July-September 1994 (subsets SET = 108-110, Jetsu et al. 1999b), which support the spot evolution. The July 1995 image resembles the July 1994 image, but with an additional new appendage stretched "southwards". The coolest part of the spot $(3700 \mathrm{~K})$ has regained its strength.
The best surface imaging solution was achieved with the differential rotation coefficient $\alpha=-0.17$. This implies strong antisolar differential rotation in HD 199178, i.e. that the pole would rotate faster than the equator. It seems that current models for global circulation in outer stellar convection zones predict only solar-like differential rotation (cf. Kitchatinov \& Rüdiger 1999). Therefore we also wanted to test the possibility of no differential rotation and solar-like differential rotation. Our Fig. 5 displays the July 1995 surface imaging solutions obtained with 

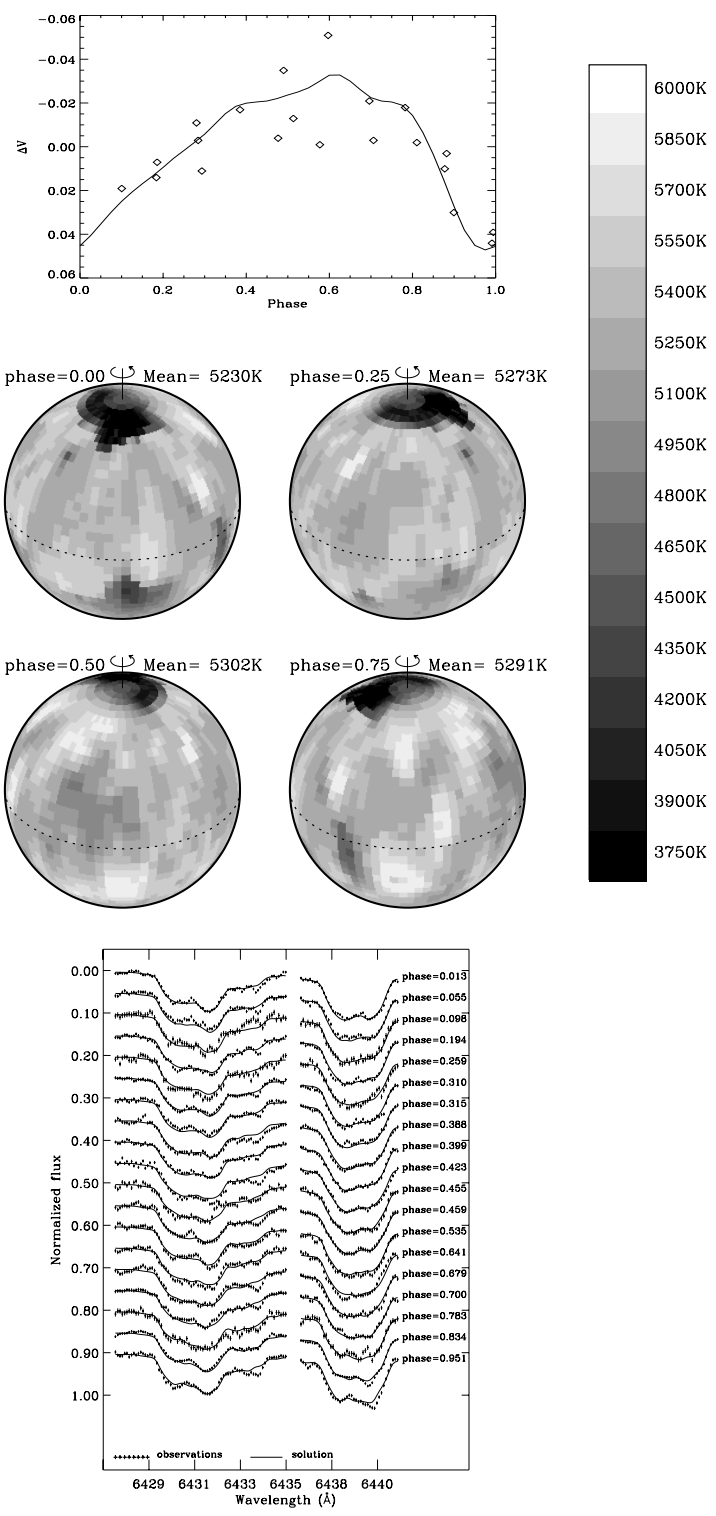

Fig. 1. The July 1994 image of HD 199178 with calculated and observed spectra and photometric $V$-curve. The mean deviation of the spectroscopic observations from the model was $d=0.56 \%$.

$\alpha=0$ (rigid body rotation) and $\alpha=0.15$ (solar-like differential rotation). The best fits with $\alpha=0$ and $\alpha=0.15$ were achieved by increasing $v \sin i$ to $72 \mathrm{~km} \mathrm{~s}^{-1}$ and $74 \mathrm{~km} \mathrm{~s}^{-1}$, respectively. The mean deviation $(d)$ of the calculated line profiles from the observations was slightly higher for $\alpha=0$ and $\alpha=0.15$ than for $\alpha=-0.17$, i.e. the antisolar case provided a better fit to the data (Table 5).

The images in Fig. 5 demonstrate the difficulties in separating the effect of a polar spot from that of differential rotation. With no differential rotation the polar region is $\sim 800 \mathrm{~K}$ cooler than the mean surface temperature, while $\alpha=0.15$ gives a cool polar spot with $T_{\text {eff }} \approx 3900 \mathrm{~K}$, much
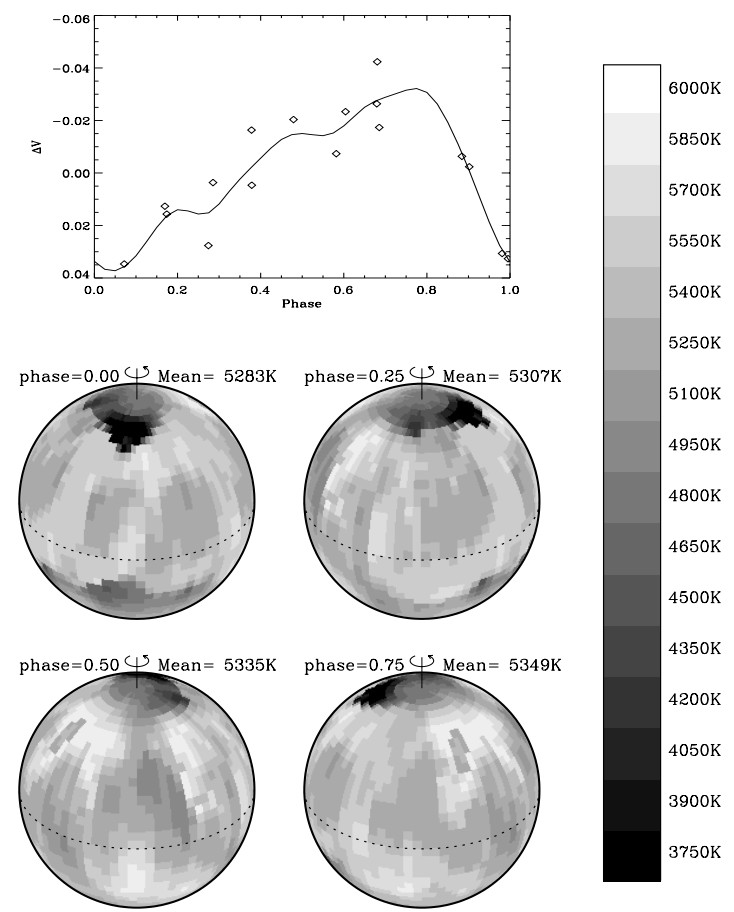

K

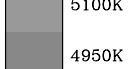

$4800 \mathrm{~K}$

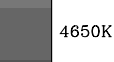

$4500 \mathrm{~K}$

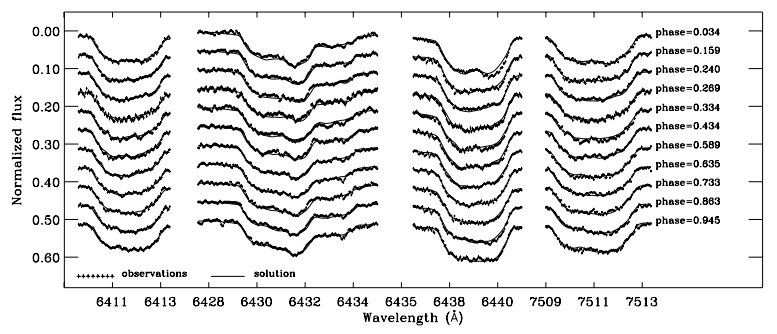

Fig. 2. The August 1994 image of HD 199178 with calculated and observed spectra and photometric $V$-curve. The mean deviation of the spectroscopic observations from the model was $d=0.43 \%$.

Table 3. Stellar parameters used for the surface images in Figs. 1-4.

\begin{tabular}{lc}
\hline Parameter & Adopted value \\
\hline Gravity $\log (g)$ & 3.5 \\
Inclination $i$ & $60^{\circ}$ \\
Rotation velocity $v \sin i$ & $70 \mathrm{~km} \mathrm{~s}^{-1}$ \\
Differential rotation $\alpha$ & -0.17 \\
Rotation period $P_{\text {rot }}$ & 3.3250 \\
Micro turbulence $v_{\text {micro }}$ & $1.4 \mathrm{~km} \mathrm{~s}^{-1}$ \\
Macro turbulence $v_{\text {macro }}$ & $4.0 \mathrm{~km} \mathrm{~s}^{-1}$ \\
Element abundances & solar \\
\hline
\end{tabular}

like the results by Strassmeier et al. (1999). The same general effect was verified for all other seasonal images. Except for the polar cap, the images in Fig. 5 are very similar. The coolest part of the spot remains at approximately the same position. Similar artifacts can also be 


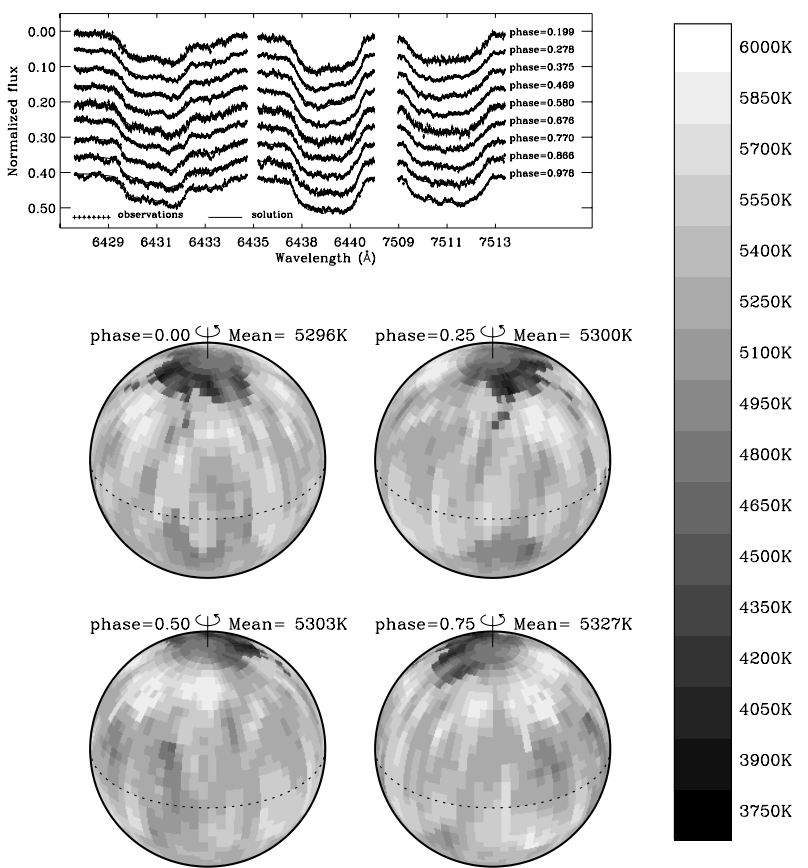

Fig. 3. The November 1994 image of HD 199178 with calculated and observed spectra (no $V$-photometry available). The mean deviation of the spectroscopic observations from the model was $d=0.50 \%$.

Table 4. Temperatures $T_{\text {spot }}$, longitudes $\ell$ and latitudes $b$ of the coolest concentrated parts of the spot in each image, and photometric periods $P$ for each set from Jetsu et al. (1999b). (The photometric periods are listed only for comparison, each image was derived using the same photometric period $P=$ 3.3250 , i.e. the mean period for the years 1994-1995.) At phase $\phi$ the longitude $\ell=-360^{\circ} \phi$ is at the center of the visible stellar disk.

\begin{tabular}{lcccl}
\hline Image & $T_{\text {spot }}(\mathrm{K})$ & $\ell\left(^{\circ}\right)$ & $b\left(^{\circ}\right)$ & $P(\mathrm{~d})$ \\
\hline July 1994 & 3700 & -5 & 67 & 3.352 \\
August 1994 & 3700 & -15 & 61 & 3.384 \\
November 1994 & 4100 & -70 & 65 & - \\
July 1995 & 3700 & -39 & 68 & 3.2829 \\
\hline
\end{tabular}

seen in all three images, in particular some bright features and smaller spots near the equator.

\section{Differential rotation or polar spot?}

The surface imaging solution with antisolar surface differential rotation might raise the question of whether this is a real phenomenon or just a trick to eliminate a cool polar spot. Thus, what we need is a way to determine which one, the polar spot or differential rotation, gives a better solution. When analyzing the T Tauri star Sz 68,
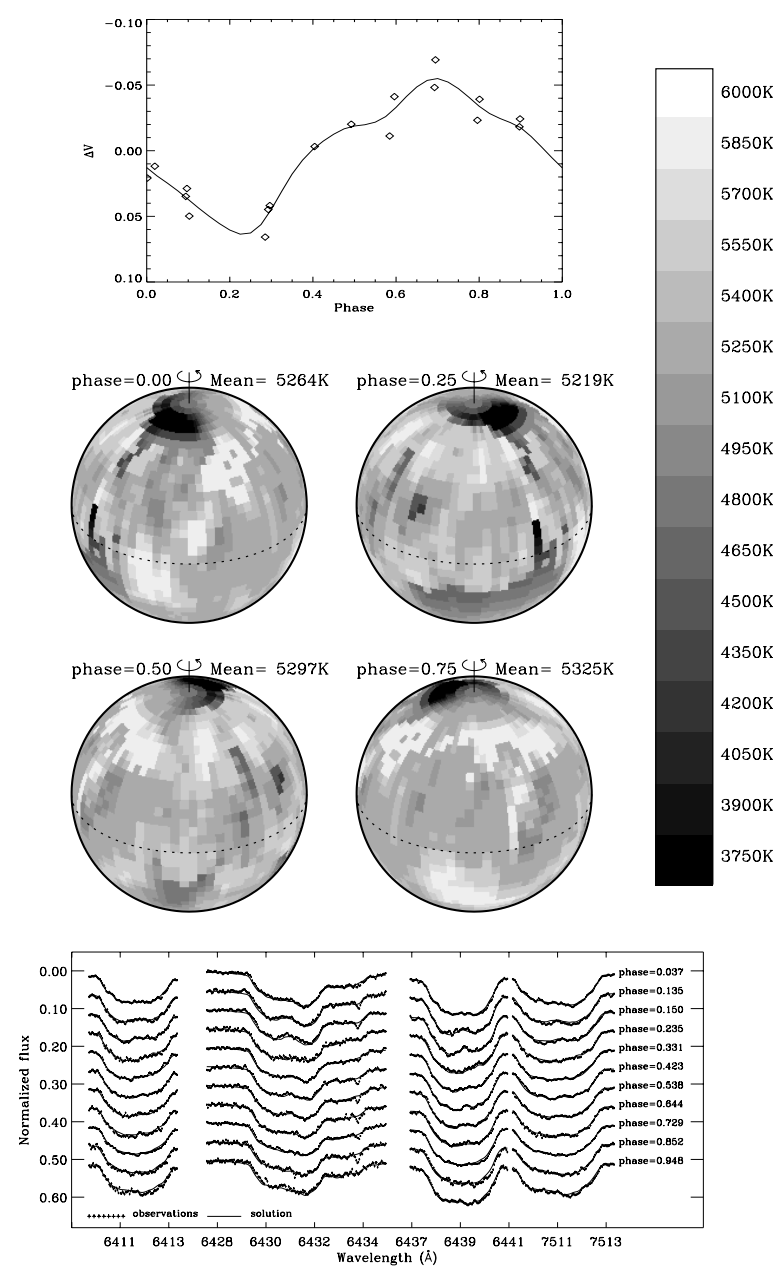

Fig. 4. The July 1995 image of HD 199178 with calculated and observed spectra and photometric $V$-curve. The mean deviation of the spectroscopic observations from the model was $d=0.37 \%$.

Table 5. The quality of the July 1995 surface imaging solution using different values of $\alpha$ : mean deviation $d$ of the calculated line profiles from the spectroscopic observations and linear correlation coefficient $r$ between next-to-neighbouring residuals.

\begin{tabular}{crcc}
\hline$v \sin i$ & $\alpha$ & $d$ & $r$ \\
\hline $70 \mathrm{~km} \mathrm{~s}^{-1}$ & -0.17 & $0.373 \%$ & 0.507 \\
$72 \mathrm{~km} \mathrm{~s}^{-1}$ & 0.00 & $0.388 \%$ & 0.540 \\
$74 \mathrm{~km} \mathrm{~s}^{-1}$ & 0.15 & $0.410 \%$ & 0.572 \\
\hline
\end{tabular}

Johns-Krull \& Hatzes (1997) tackled this problem by calculating separate temperature maps for different spectral lines. They argued for solid body rotation and a polar spot, because this alternative gave a larger correlation between the individual maps.

We tested whether this approach works in our case. First we simulated synthetic spectroscopic "observations" using as input a stellar surface with a single high latitude 


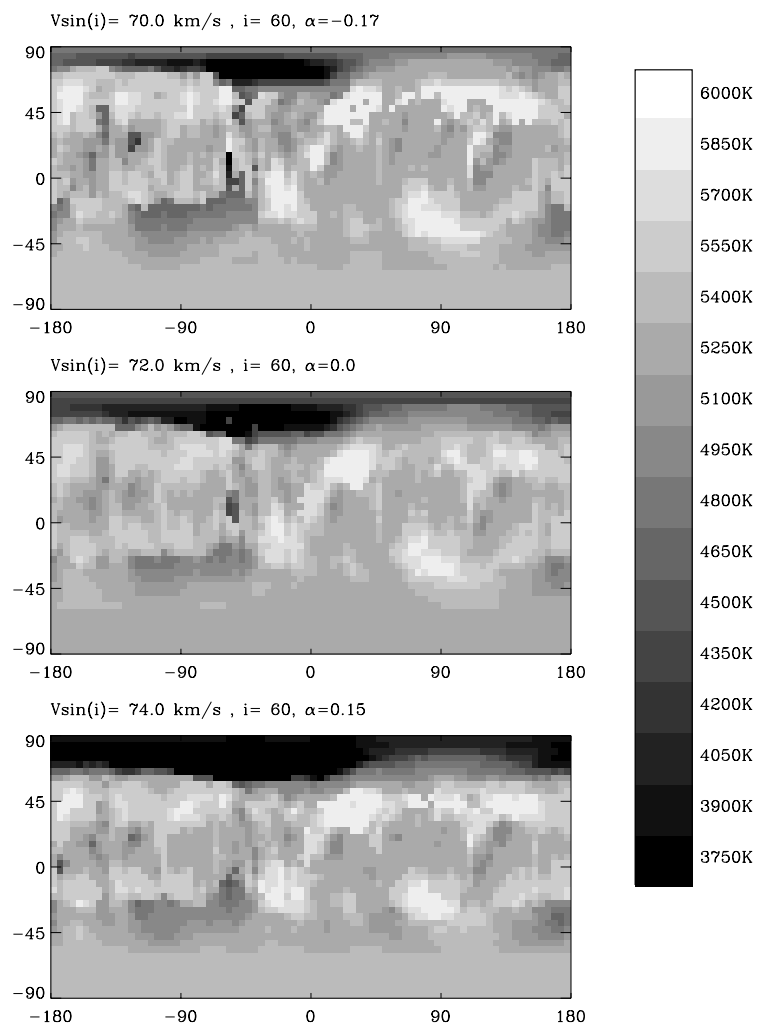

Fig. 5. The July 1995 image with differential rotation coefficients $\alpha=-0.17$ (same as Fig. 4), 0.0 and 0.15 .

spot, but no polar spot, and stellar parameters $v \sin i=$ $70 \mathrm{~km} \mathrm{~s}^{-1}, \alpha=-0.2, i=60^{\circ}$ (CASE I). To imitate real observations, we added normally distributed noise $(\sigma=0.30 \%)$ to the synthetic observations. These synthetic spectra were then used to calculate temperature maps with two sets of stellar parameters: $v \sin i=$ $70 \mathrm{~km} \mathrm{~s}^{-1}, \alpha=-0.2, i=60^{\circ}$ and $v \sin i=72 \mathrm{~km} \mathrm{~s}^{-1}$, $\alpha=0.0, i=60^{\circ}$. Maps were calculated both using all spectral regions and separately for each spectral region (6411 $\AA$, $6431 \AA, 6439 \AA$ and $7511 \AA$ ). With the correct stellar parameters, we retrieved a surface imaging solution consistent with the input stellar surface. As expected, neglecting the differential rotation, i.e. using $\alpha=0.0$, resulted in a solution with a cool polar spot in addition to the high latitude spot.

For both sets of stellar parameters we calculated the correlations between the maps retrieved for the different spectral lines. The test results showed that the correlation was higher when the differential rotation was wrongly neglected (Tables 6 and 7). The reason for this is clear. Neglecting a substantial differential rotation, one always obtains the same artifact, a polar spot, regardless of the spectral line used for the image. The presence of the same polar spot in all images will naturally increase the correlation between the images. The correlation only
Table 6. CASE I: correlation between solutions for different spectral lines. The solutions were retrieved neglecting differential rotation $(\alpha=0)$, although the original data was simulated with a differential rotation coefficient $\alpha=-0.2$.

\begin{tabular}{lllll}
\hline & $6411 \AA$ & $6431 \AA$ & $6439 \AA$ & $7511 \AA$ \\
\hline $6411 \AA$ & $r=1.000$ & & & \\
$6431 \AA$ & $r=0.975$ & $r=1.000$ & & \\
$6439 \AA$ & $r=0.959$ & $r=0.967$ & $r=1.000$ & \\
$7511 \AA$ & $r=0.961$ & $r=0.953$ & $r=0.970$ & $r=1.000$ \\
\hline
\end{tabular}

Table 7. CASE I: correlation between solutions for different spectral lines. Both the solutions and the original data were simulated with the differential rotation coefficient $\alpha=-0.2$.

\begin{tabular}{lllll}
\hline & $6411 \AA$ & $6431 \AA$ & $6439 \AA$ & $7511 \AA$ \\
\hline $6411 \AA$ & $r=1.000$ & & & \\
$6431 \AA$ & $r=0.899$ & $r=1.000$ & & \\
$6439 \AA$ & $r=0.918$ & $r=0.907$ & $r=1.000$ & \\
$7511 \AA$ & $r=0.887$ & $r=0.913$ & $r=0.892$ & $r=1.000$ \\
\hline
\end{tabular}

measures the consistency between the calculated images. It will not reveal the inconsistency between the original data (no polar spot) and the solution (polar spot).

An alternative approach to check the reliability of Doppler imaging maps, is to estimate the systematic errors in the calculated line profiles. As opposed to noise, the systematic errors of neighbouring wavelength points will correlate. In other words, systematic errors will cause the neighbouring data points to tend to be on the same side of the calculated curve. Not surprisingly, the linear correlation coefficient $r$ of neighbouring residuals, i.e. the systematic error, was significantly larger for the solution with the (false) solid body rotation than for the one with the (correct) differential rotation (Table 8: 1st and 2nd line). The systematic error could also be seen in the slightly larger deviation $d$.

To show that this criterion (lowest correlation of residuals) does not favour any particular spot distribution, we also simulated synthetic observations with a polar spot and no differential rotation (CASE II). Again the simulated data was used to retrieve temperature maps with and without differential rotation. The correct stellar parameters gave a solution consistent with the input map. In the solution with artificial differential rotation the polar spot was reduced, as expected. But also in this case, an erroneous differential rotation coefficient caused correlation in the residuals and increased the deviation (Table 8: 3rd and 4th line).

We conclude, that aiming for a maximum correlation between images obtained from different spectral lines will favour solutions with polar spots. Furthermore, systematical errors in the calculated line profiles increase, if the value of $\alpha$ is erroneous.

In the simulated tests we correlated the errors of neighbouring points. But in our real data of HD 199178 neighbouring residuals will anyway be correlated, because the 
Table 8. The quality of the surface imaging solution for rigid body $(\alpha=0)$ and antisolar differential rotation $(\alpha=-0.2)$ in CASES I and II: mean deviation $d$ of the solution from the synthetic spectroscopic "observations" and linear correlation $r$ of subsequent residuals of the line profiles.

\begin{tabular}{lcr|crccc}
\hline \multicolumn{2}{c|}{ Input parameters } & \multicolumn{3}{|l}{ Solution } \\
polar spot & $v \sin i$ & $\alpha$ & $v \sin i$ & $\alpha$ & $d$ & $r$ \\
\hline no (CASE I) & 70 & -0.2 & 70 & -0.2 & $0.30 \%$ & 0.035 \\
no (CASE I) & 70 & -0.2 & 72 & 0.0 & $0.33 \%$ & 0.198 \\
\hline yes (CASE II) & 72 & 0.0 & 70 & -0.2 & $0.32 \%$ & 0.110 \\
yes (CASE II) & 72 & 0.0 & 72 & 0.0 & $0.30 \%$ & -0.001 \\
\hline
\end{tabular}

spectra have been rebinned to a common wavelength scale. This effect can be avoided by comparing each point in the spectra to the point two steps ahead. The correlation of next-to-neighbouring residuals was checked for the July 1995 surface imaging solutions with the differential coefficients $\alpha=-0.17, \alpha=0.0$ and $\alpha=0.15$ (Fig. 5).

The results in Table 5 showed that the solution with $\alpha=-0.17$ gave both the best fit (lowest $d$ ) and smallest correlation of the residuals (lowest $r$ ). However, we note that the correlation was highly significant even for the best stellar parameters $\left(v \sin i=70 \mathrm{~km} \mathrm{~s}^{-1}, \alpha=-0.17\right.$, $i=60^{\circ}$ ). Apparently there still remained systematic errors in the solution. This could also be seen from the fact that the deviation was larger than the observational noise. The most likely cause for these systematic errors were inaccuracies in the calculated line profiles (missing lines, errors in spectral parameters or element abundances, non-LTE effects, etc.). Even though all systematic errors could not be eliminated, we consider negative surface differential rotation more likely than the polar spot, because the best quality of the surface imaging solution was achieved with $\alpha=-0.17$. Still, we emphasize that the $\alpha$-value was used to adjust the calculated profiles to fit the observations. Therefore our estimate for $\alpha$ may be biased by errors in the line calculations.

The presence of differential rotation is supported by the variation of the photometric period, although photometry alone can only give a lower limit for $|\alpha|$. Inserting the means of the spot latitudes and photometric periods of Table 4 into Eq. (4), the value of $\alpha=-0.17$ would give the rotation periods at the equator $P_{\text {equator }} \approx 3.81$ and the pole $P_{\text {pole }} \approx 3$ d 25 . The reliable photometric periods of HD 199178 determined by Jetsu et al. (1999b) were within the range $3.191 \pm 0.017 \leq P_{\text {phot }} \leq 3^{\mathrm{d}} .559 \pm 0.017$. Since HD 199178 is a rapid rotator, one would expected it to have only high latitude spots (Schüssler \& Solanki 1992). Therefore it is natural, that we do not find the equatorial rotation period from photometry. But the fact that our $P_{\text {pole }}$ is larger than the smallest measured $P_{\text {phot }}$ indicates a slight discrepancy.

By combining the time series analysis of photometry with individual surface images we can study if the photometric rotation period depends on the latitude of the main spot. Antisolar differential rotation would be seen as a shorter period for higher latitude spots. We note that this is indeed the case for the photometric periods and latitudes of the spot in Table 4. But since we have only three measurements, this tendency is far from statistically significant. Furthermore, the differences in the spot latitudes obtained from the surface images are too small to draw reliable conclusions.

There are other methods to measure the surface differential rotation. The approach most commonly used in Doppler imaging, is to cross-correlate constant latitude slices of images and calculate the shift in longitude as a function of the latitude (cf. Donati \& Collier Cameron 1997). This approach requires that there really are longlived spots at different latitudes. The method may also fail if there are too many artifacts in the images. Artifacts will often appear at nearly the same longitude as the real features or as vertical stripes in the images. A crosscorrelation analysis of such images will give a nearly rigid body rotation. In our images, there is only one major spot, and the low latitude features are probably artifacts. For this reason, we do not attempt to cross-correlate our images.

Antisolar differential rotation has previously been found in some active late-type stars. Johns-Krull (1996) analyzed the rotationally broadened spectral line profiles of three T Tauri stars and got the best fits with antisolar differential rotation. But he also noted that a polar spot could produce this effect. Weak antisolar differential rotation has been found in Doppler images of the RS CVn stars UX Ari (Vogt \& Hatzes 1996), HR 1099 (Vogt et al. 1999) and HD 106225 (Strassmeier 1994; Hatzes 1998).

\section{Conclusions}

The surface images of HD 199178 presented here resemble the results recently obtained for FK Comae Berenices (Korhonen et al. 1999, 2000). The dominating feature is a long-lived high latitude spot (or spot group), with slightly changing position, shape and strength. Such changes could already be seen in the July 1994 and August 1994 images. In the November 1994 image, the spot had weakened and partly disintegrated. In July 1995, the spot had recovered its strength. The changes in the photometric light curves of HD 199178 during 1994 and 1995 can thus be explained by evolution of a single large spot rather than by abrupt changes of the whole spot configuration.

The long-lived spot structure suggests there is a stable active longitude in HD 199178. The high latitude of the spot could be explained by that the Coriolis force dominates over the buoyancy force in the magnetic flux tubes erupting from the deep parts of the convection zone (Schüssler \& Solanki 1992). The changes in the spot latitude could then be connected to variations in the magnetic field strength. The changes in the latitude will in turn lead to drifts in the longitude if there is surface differential rotation. 
In previous temperature maps of HD 199178, the "flat bottomed" absorption line profiles have been interpreted as a signature of a large and very cool polar spot (Vogt 1988; Strassmeier et al. 1999). We achieved the best fit to the spectral observations by introducing an antisolar surface differential rotation of $\alpha=-0.17$. With $\alpha \geq 0$, a polar spot appeared in the images. We considered the solution with antisolar differential rotation to be more likely than the polar spot, because it reduced both the discrepancy between the observed and calculated line profiles, as well as the correlation between residuals of neighbouring wavelength points (i.e. systematical errors in the calculated line profiles).

During 1994 and 1995 the photometric rotation period varied by $3 \%$. Jetsu et al. (1999b) interpreted these variations as a signature of surface differential rotation. Our images show that the photometric minimum of the light curves was apparently caused by the same persistent high latitude spot (group). Therefore the changes in the photometric period could not be explained by a changing pattern of spots. We also note that in our surface images the changes in the spot latitude were less than 10 degrees. The differential rotation would have to be strong in order to cause the observed changes in the photometric rotation period.

There are certainly other alternative explanations for the flat bottomed line profile shape than antisolar differential rotation. The very rapid rotation of FK Comaetype stars, perhaps being recently coalesced binaries, suggests there could be significant deviations from a spherical shape (cf. Weltey \& Ramsey 1994). Our understanding of absorption line formation in the photosphere of these exceptional stars may also be insufficient. In particular, the strong absorption lines may be subject to non-LTE effects. On the other hand, Bruls et al. (1998) showed that chromospheric activity and non-LTE effects could only explain the box-like shape of some (e.g. Fe I $\lambda 6430 \AA$ ), but not all (e.g. Fe I $\lambda 6411 \AA$ and Ca I $\lambda 6439 \AA$ ) of the lines used in our analysis.

Weak antisolar differential rotation has previously been detected in some RS CVn stars (cf. Vogt et al. 1999). These detections were made by comparing Doppler images having a time difference of up to several months. This approach is, however, only possible for stars with a very small differential rotation, since the largest sensible phase difference is 0.5 . Secondly, it is hard to confirm that the same particular spots are really present in different images taken months apart.

Strassmeier et al. (1999) cross-correlated constant latitude slices of images to determine the differential rotation of HD 199178. They could not see any effects of differential rotation but stated that this "non-detection does not necessarily mean there is no differential surface rotation". We stress that the cross-correlation will provide reliable results only if there are long-lived spots on different latitudes. That is not the case in our images of HD 199178. Furthermore, the result may be biased by artifacts in the images.
Differential rotation can also be detected by combining Doppler images and time series analysis of photometry. If there is a clear correlation between the latitude of the main spot and the photometric period, one could determine the law of the differential rotation. But this kind of analysis would require more data than was available for the present study.

Acknowledgements. The work of T.H. was supported by a grant from the Jenny and Antti Wihuri foundation, and by Helsinki University research funding for the project "Time series analysis in astronomy" (No. 974/62/98). I. Ilyin reduced the July 1994 spectroscopic data, participated in the observations and gave useful comments on the manuscript. The molecular line data were kindly supplied by S. Berdyugina. We also thank the anonymous referee, whose suggestions helped to improve the paper. The surface imaging temperature maps were calculated using the Cray C94/128 supercomputer at the Centre for Scientific Computing, Espoo, Finland. This research has made use of the Simbad-database operated at CDS, Strasbourg, France.

\section{References}

Berdyugina, S. V. 1991, Izv. Krymsk. Astrofiz. Obs., 83, 102 Berdyugina, S. V. 1998, A\&A, 338, 97

Bopp, B. W., \& Rucinski, S. M. 1981, in Fundamental Problems in the Theory of Stellar Evolution, IAU Symp. 93, ed. D. Sugimoto, D. Q. Lamb, \& D. N. Schramm (Reidel, Dordrecht), 177

Bruls, J. H. M. J., Solanki, S. K., \& Schüssler, M. 1998, A\&A, 336,231

Bruning, D. H. 1981, ApJ, 248, 274

Cutispoto, G. 1998, A\&AS, 131, 321

Donahue, R. A., Saar, S. H., \& Baliunas, S. L. 1996, ApJ, 466, 384

Donati, J.-F., \& Collier Cameron, A. 1997, MNRAS, 291, 1

Hall, D. S., 1991, in The Sun and Cool Stars, IAU Coll. 130, ed. I. Tuominen, D. Moss, \& G. Rüdiger (Springer-Verlag, Heidelberg), 353

Hatzes, A. P. 1998, A\&A, 330, 541

Hatzes, A. P., \& Vogt, S. S. 1992, MNRAS, 258, 387

Hatzes, A. P., Vogt, S. S., Ramseyer, T. F., \& Misch, A. 1996, ApJ, 469, 808

Herbig, G. H. 1958, ApJ, 128, 259

Ilyin, I. V. 2000, High resolution SOFIN CCD échelle spectroscopy, Academic Dissertation, University of Oulu

Jetsu, L., Huovelin, J., Tuominen, I., et al. 1990, A\&A, 236, 423

Jetsu, L., Tuominen, I., Bopp, B. W., et al. 1999a, A\&AS, 139, 513

Jetsu, L., Pelt, J., \& Tuominen, I. 1999b, A\&A, 351, 212

Johns-Krull, C. M. 1996, A\&A, 306, 803

Johns-Krull, C. M., \& Hatzes, A. P. 1997, ApJ, 487, 896

Kitchatinov, L. L., \& Rüdiger, G. 1999, A\&A, 344, 911

Korhonen, H., Berdyugina, S. V., Hackman, T., et al. 1999, A\&A, 346, 101

Korhonen, H., Berdyugina, S. V., Hackman, T., et al. 2000, A\&A, 360, 1067

Kupka, F., Piskunov, N. E., Ryabchikova, T. A., et al. 1999, A\&AS, 138, 119

Kurucz, R. L. 1993, ATLAS-9, CD-ROM \#13 
Lacy, C. H. 1977, ApJS, 34, 479

Perryman, M. A. C., Lindegren, L., Kovalevsky, J., et al. 1997, A\&A, 323, L49

Piskunov, N. E. 1991, in The Sun and Cool Stars, IAU Coll. 130, ed. I. Tuominen, D. Moss, \& G. Rüdiger (SpringerVerlag, Heidelberg), 309

Piskunov, N. E., Tuominen, I., \& Vilhu, O. 1990, A\&A, 230, 363

Schüssler, M., \& Solanki, S. K. 1992, A\&A, 264, L13

Strassmeier, K. G. 1994, A\&A, 281, 395

Strassmeier, K. G., Hall, D. S., Boyd, L. J., \& Genet, R. M. 1989, ApJS, 69, 141

Strassmeier, K. G., Rice, J. B., Wehlau, W. H., et al. 1991, A\&A, 247, 130

Strassmeier, K. G., Lupinek, S., Dempsey, R. C., \& Rice, J. B.
1999, A\&A, 347, 212

Straižys, V., Goldberg, E. P., Meištas, E., \& Vansevičius, V. 1989, A\&A, 222, 82

Unruh, Y. C., \& Collier Cameron, A. 1995, MNRAS, 273, 1

Vogt, S. S. 1988, in The Impact of Very High $S / N$ Spectroscopy on Stellar Physics, IAU Symp. 132, ed. G. Cayrel de Strobel, \& M. Spite (Kluwer Academic Publishers, Dordrecht), 253

Vogt, S. S., Hatzes, A. P. 1991, in The Sun and Cool Stars, IAU Coll. 130, ed. I. Tuominen, D. Moss, \& G. Rüdiger (Springer-Verlag, Heidelberg), 297

Vogt, S. S., Hatzes, A. P., Misch, A. A., \& Kürster, M. 1999, ApJS, 121,547

Weltey, A. D., \& Ramsey, L. W. 1994, ApJ, 435, 848 